\title{
Extracellular Fluid and Total Body Water Changes in Neonates Undergoing Extracorporeal Membrane Oxygenation
}

\author{
By Harry L. Anderson III, Arnold G. Coran, Robert A. Drongowski, Hyun J. Ha, and Robert H. Bartlett \\ Ann Arbor, Michigan
}

\begin{abstract}
- After being placed on extracorporeal life support (ECLS), newborn patients typically weight $5 \%$ to $30 \%$ more than their birthweight. Recovery and eventual decannulation from ECLS is associated with a return to baseline weight or birthweight values after a pronounced diuresis. It has been assumed that the increases in weight in these patients are due to increases in extracellular fluid (ECF) and total body water (TBW). This study was undertaken to prove or disprove this hypothesis. ECF space was measured using the compound sodium bromide and TBW was determined with the use of deuterium oxide (nonradioactive heavy water). Fluid compartment measurements were made prior to the institution of ECLS, immediately after placement on bypass, approximately every other day while on bypass, and a final measurement was made once the patient was off bypass. Sodium bromide concentration was analyzed by highpressure liquid chromatography, and deuterium oxide concentration was measured by the falling drop method. Eight newborns with respiratory failure were placed on either venoarterial (4 patients) or venovenous (4 patients) ECLS for an average of 106 hours (range, 71 to 219 hours). Pre-ECLS TBW was high in the neonates $(87 \%$ of total body weight $v$ the normal of $75 \%$ to $80 \%$ ). Mean values for each fluid compartment were corrected for the additional volume of the bypass circuit when the patient was on bypass. ECF increased immediately after the institution of ECLS; however, both ECF and TBW decreased during the bypass run, and post-ECLS levels of ECF and TBW were similar to those found prior to ECLS. Body weight increased significantly at the onset of ECLS and returned to near pre-ECLS (birthweight) levels at the conclusion of bypass. We conclude that neonates with respiratory distress, once placed on ECLS, have increased body weight, ECF, and TBW, which decrease during the course of ECLS, and reach baseline levels. These decreases in weight, ECF, and TBW appear to be associated with lung recovery.

Copyright $\odot 1992$ by W.B. Saunders Company
\end{abstract}

INDEX WORDS: Extracorporeal membrane oxygenation (ECMO): extracorporeal life support: neonatal respiratory failure; extracellular fluid space; total body water.

$\mathbf{E}^{\mathrm{X}}$ XTRACORPOREAL membrane oxygenation (ECMO), or more accurately, extracorporeal life support (ECLS), is accepted therapy for newborns with the neonatal respiratory failure syndrome. We have noted an increase of $5 \%$ to $30 \%$ in body weight of these newborns with respiratory failure over birthweight after cannulation for ECLS. A diuresis to baseline weight or birthweight usually is followed by lung recovery and, shortly thereafter, decannulation from bypass. This increase in body weight after cannulation has been attributed to increases in the extracellular fluid space (ECF space) and total body water (TBW). To investigate this hypothesis, we measured the ECF and TBW before, during, and after ECLS.

\section{MATERIALS AND METHODS}

Eight newborn patients, either born at the University of Michigan Medical Center or referred from other institutions to our medical center, were entered into this study. All neonates had significant respiratory failure unresponsive to conventional medical management, and thereby met criteria for ECLS using standard parameters. ${ }^{1}$ The need for urgent cannulation (hypotension, significant hypoxemia/hypercarbia/acidosis) were considered as contraindications for entry into this study. Bypass was conducted with standard venoarterial (jugular vein to carotid artery) or double lumen, single catheter venovenous (jugular vein) access. ${ }^{1}$ The measurement of ECF and TBW compartments in neonates with respiratory failure was approved by the Institutional Review Board of the University of Michigan.

Informed consent for this study was obtained from either parent prior to the institution of ECLS. Blood sampling ( $6 \mathrm{~mL}$ was the approximate volume withdrawn each measurement day) was not directly replaced, and sampling was performed via indwelling catheters (umbilical vessel catheters) or via access lines of the extracorporeal bypass circuit. Blood sampling for measurement of ECF and TBW was carried out prior to and just after the institution of ECLS, after 1 day of ECLS, and then approximately every other day for the next 5 days during ECLS. One single measurement was made after decannulation. The techniques of ECF space measurement using sodium bromide ${ }^{2}$ and TBW measurement using deuterium oxide ${ }^{3}$ have been previously described. Briefly, each measurement was performed by first drawing an initial sample of blood. Known amounts of sodium bromide (5\% solution in sterile water, 2 $\mathrm{mL} / \mathrm{kg}$ body weight) and deuterium oxide (nonradioactive heavy water, $1.5 \mathrm{~mL} / \mathrm{kg}$ body weight) were then slowly administered to the patient by intravenous infusion over 10 to 20 minutes. Equilibration of these tracers was then allowed for approximately 120 minutes, at which time a second sample was collected. Samples were immediately centrifuged at $2,000 \mathrm{rpm}$ at $0^{\circ} \mathrm{C}$ for 15 minutes, and the supernatants were individually stored by freezing for later assay.

ECF space was determined by measuring bromide concentration in the supernatant samples using high-pressure liquid chromatography. TBW was determined by measuring deuterium oxide concentration in the supernatant by the falling drop method after

From the Section of Pediatric Surgery, C.S. Mott Children's Hospital, University of Michigan Medical Center, Ann Arbor, MI.

Supported in part by grants from the National Institutes of Health and the Clinical Research Center of the University of Michigan.

Presented at the Jens $G$. Rosencranz Resident Competition at the 43rd Annual Meeting of the Surgical Section of the American Academy of Pediatrics, New Orleans, Louisiana, October 26-27, 1991.

Address reprint requests to A.G. Coran, $M D$, Section of Pediatric Surgery, L2110 Matemal-Child Health Center, University of Michigan Medical Center, Ann Arbor, MI 48109-0245.

Copyright 11992 by W.B. Saunders Company

$0022-3468 / 92 / 2708-0012 \$ 03.00 / 0$ 
double-vacuum distillation of the supernatant. Using the patient's daily weight and the resultant measured tracer concentration after administration of known amounts of the tracer compounds, ECF and TBW values were calculated using previously described equations. ${ }^{2,3}$ During ECLS bypass, calculations involving patient weight were corrected, based on the sum of the patient's weight and the weight (by volume analysis) of the blood contained within the extracorporeal circuit. ECF and TBW volumes, also based on the corrected patient weight, were calculated before, during, and after ECLS.

Patient weight, ECF space, and TBW percentages prior to bypass were compared with values: (1) upon initiation of bypass; (2) just prior to the conclusion of bypass; and (3) after decannulation from bypass. Statistical significance was determined using the unpaired Student's $t$ test of two means. A $P<.05$ was considered statistically significant.

\section{RESULTS}

Demographics of the eight patients are listed in Table 1. One patient (case 1) was taken to the operating room after decannulation from ECLS for correction of a cardiac anomaly by a Blalock-Taussig operation.

ECF and TBW measurements of the eight neonates are displayed in Fig 1 and 2, respectively. Values for ECF and TBW are expressed as a percentage of corrected body weight. Patient weight during ECLS was corrected by adding $0.63 \mathrm{~kg}$ to the measured patient weight to account for the weight of the blood volume within the extracorporeal circuit. TBW decreased after initiation of bypass and showed a continuous decrease until just prior to decannulation, at which time a statistically significant nadir (compared with pre-ECLS) was reached. In the postECLS period, TBW was below baseline, although this difference was not statistically significant.

In Fig 3, the mean of corrected patient weights during the bypass course is displayed. Again, patient weights during bypass represent the measured patient weight plus an additional $0.63 \mathrm{~kg}$ to account for the weight of the extracorporeal blood volume. By 24 hours on bypass (day 1), mean corrected weight reached a peak of $4.5 \mathrm{~kg}$ over the pre-ECLS patient weight of $3.4 \mathrm{~kg}$. Corrected patient weight continued to fall during the remainder of the ECLS course. Patient weights pre-ECLS and post-ECLS were not significantly different.

Also displayed in Fig 3 are the means of the calculated ECF and TBW volumes. The calculated ECF and TBW volumes each increased on initiation of bypass, and decreased below the pre-ECLS baseline during the course of ECLS. The calculated ECF and TBW volumes were similar pre-ECLS and postECLS. The ECF volume reached a maximum after 24 hours on ECLS (day 1), while the TBW volume reached a maximum just after initiation of bypass (day 0).

The absolute values of ECF, TBW, and uncorrected patient weight prior to, after the initiation of, prior to the conclusion of, and after ECLS are displayed in composite form in Table 2. Weight upon initiation of ECLS and prior to the conclusion of ECLS were significantly higher than pre-ECLS. PreECLS and post-ECLS weights were not significantly different.

\section{DISCUSSION}

Several investigators have followed changes in the body fluid compartments in humans ${ }^{4}$ and animals ${ }^{5-8}$ before, during, and after extracorporeal circulation of blood. The primary focus of these studies was the effects of short-term bypass (eg, cardiopulmonary bypass for intracardiac surgery) and hypothermia on body fluid compartments. Brans et al ${ }^{6}$ used neonatal baboons treated with ECMO for 7 to 10 hours and showed no statistically significant changes in TBW, extracellular water, and plasma volume.

We have noted increases of $5 \%$ to $30 \%$ in body weight over baseline or birthweight in newborns with respiratory failure initially after placement on ECLS. These increases have been thought to be due to the accumulation of fluid as a result of the initial resuscitation prior to cannulation and intrinsic increases in extracellular and intracellular water. An increased capillary leak, resulting in ECF and/or TBW in-

Table 1. Demographics of Eight Newborn Patients With Respiratory Failure Undergoing Measurement of ECF and TBW

\begin{tabular}{|c|c|c|c|c|c|c|c|}
\hline $\begin{array}{l}\text { Case } \\
\text { No. }\end{array}$ & Diagnoses & $\begin{array}{c}\text { Gestational } \\
\text { Age (wk) }\end{array}$ & Sex & $\begin{array}{l}\text { Bypass } \\
\text { Mode }\end{array}$ & $\begin{array}{c}\text { Birth- } \\
\text { Weight (kg) }\end{array}$ & $\begin{array}{c}\text { ECLS Time } \\
\text { (h) }\end{array}$ & $\begin{array}{c}\text { Survived/ } \\
\text { Died }\end{array}$ \\
\hline 1 & Ebstein's anomaly & 40 & $F$ & VA & 3.8 & 113 & $\mathbf{S}$ \\
\hline 2 & MAS & 39 & $\mathbf{M}$ & VA & 3.4 & 92 & $\mathbf{S}$ \\
\hline 3 & RDS, PFC & 35 & $\mathbf{M}$ & VA & 2.8 & 86 & $\mathbf{S}$ \\
\hline 4 & PPHN, Marfan's syndrome & 40 & $F$ & VV & 4.1 & 103 & $\mathrm{~S}$ (late death) \\
\hline 5 & MAS & 43 & $F$ & VV & 3 & 71 & $\mathbf{S}$ \\
\hline 6 & PPHN, sepsis & 35 & M & VV & 2.9 & 219 & $\mathbf{S}$ \\
\hline 7 & Sepsis (viral) & 40 & $\mathbf{M}$ & VV & 3.7 & 80 & $\mathbf{S}$ \\
\hline 8 & PPHN & 40 & $\mathbf{M}$ & VA & 3.2 & 80 & $\mathbf{S}$ \\
\hline
\end{tabular}

Abbreviations: MAS, meconium aspiration syndrome; RDS, respiratory distress syndrome; PFC, persistent fetal circulation; PPHN, persistent pulmonary hypertension of the newborn; VA, venoarterial bypass; VV, venovenous bypass; $S$, survived. 


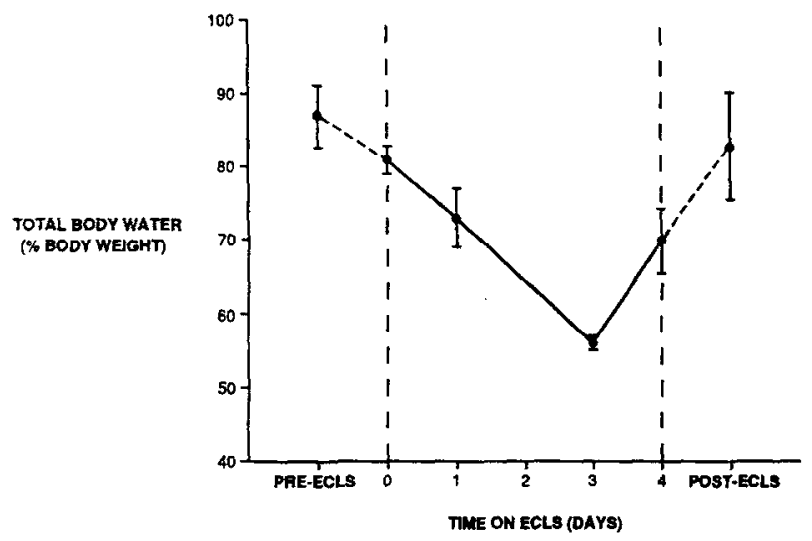

Fig 1. Changes in ECF before, during, and after ECLS bypass in $=8$ patients). ECF values listed are expressed as a percent of patient body weight.

crease, might be secondary to the altered hemodynamics of persistent fetal circulation, hypoxemia, and acidosis. Activation of blood components responsible for inflammation (complement, kinins, plasmin, prostaglandins, and cytokines) as a result of the interaction of blood with the foreign surface of the ECLS circuit results in increased capillary leak. ${ }^{1}$ The indwelling bypass cannulae may contribute only a small amount of weight $(0.02$ to $0.05 \mathrm{~kg})$ to the overall increase in patient weight during ECLS. Because excess weight in neonates on ECLS is lost through active diuresis or hemofiltration, lung healing appears to occur as the weight nears baseline or birthweight values. ${ }^{9}$ Whether loss of ECF or TBW underlies the process of lung recovery or whether lung recovery results in a diuresis, resulting in a lower ECF and TBW, is not known. Failure of ECF and TBW in reaching pre-ECLS levels as a reason for nonrecoverable lung function was not observed. All of the neonates studied achieved lung recovery.

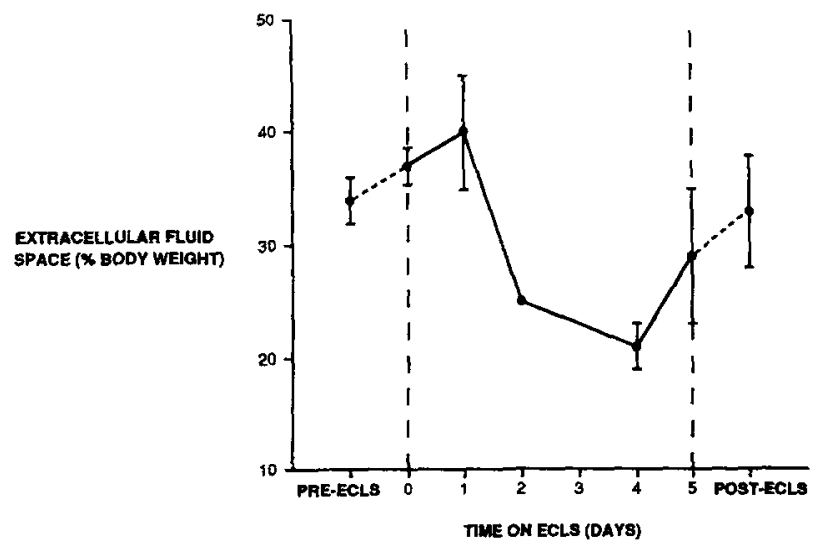

Fig 2. Changes in TBW before, during, and after ECLS bypass ( $n=7$ patients). TBW values listed are expressed as a percent of patient body weight.

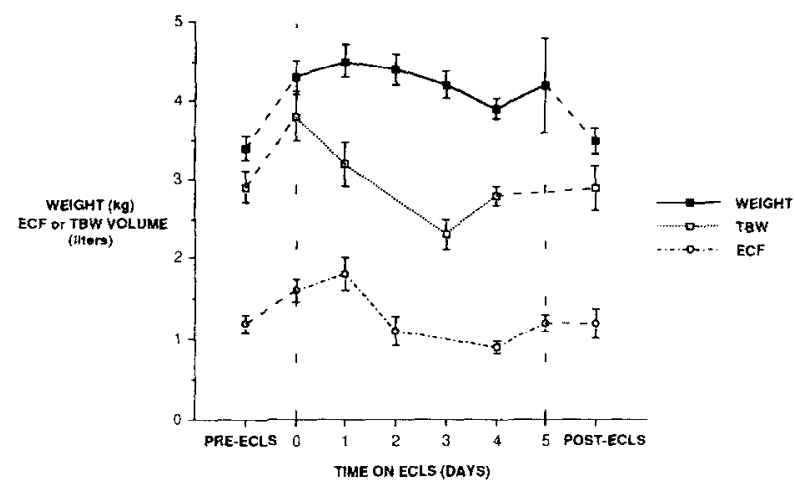

Fig 3. Changes in mean patient weight, and calculated ECF space and TBW volumes before, during, and after ECLS bypass ( $n=8$ patients). Patient weight during ECLS bypass was corrected for the presence of the extracorporeal blood volume by the addition of 0.63 kg (the weight by volume analysis of the extracorporeal circuit volume).

Direct measurement of ECF and TBW in the human neonate with respiratory failure and treated with ECLS has not been previously described. We investigated the changes in ECF and TBW prior to, during, and after ECLS was instituted for respiratory failure unresponsive to conventional medical management.

The corrected patient weight increases $1.1 \mathrm{~kg}$ over the pre-ECLS weight by day 1 , about $0.5 \mathrm{~kg}$ over and above the $0.63 \mathrm{~kg}$ factor added to account for the weight of the blood volume contained within the extracorporeal circuit. Weight continues to decrease during the bypass run, reaching the pre-ECLS baseline after decannulation. TBW decreases on initiation of bypass, which might be explained by the addition of the extracorporeal circuit volume ("prime," which includes normal saline, albumin, heparin, and packed red blood cells), combining to form a lower TBW from the already high pre-ECLS patient TBW.

The calculated TBW volume increases $0.9 \mathrm{~kg}$ upon initiation of bypass, again a change of $0.3 \mathrm{~kg}$ over the $0.63 \mathrm{~kg}$ maximum, which could theoretically be expected if the extracorporeal volume were pure water. An overall increased TBW volume probably does contribute to the increased patient weight. Since the ECF space similarly increases after the initiation of

Table 2. Comparison of Weight, ECF, and TBW in Neonates With Respiratory Failure Before, During and After ECLS

\begin{tabular}{lcccc}
\hline & Pre-ECLS & $\begin{array}{c}\text { On ECLS } \\
\text { (Initia) }\end{array}$ & $\begin{array}{c}\text { On ECLS } \\
\text { (Final) }\end{array}$ & $\begin{array}{c}\text { Post- } \\
\text { ECLS }\end{array}$ \\
\hline Weight (kg) & $3.4 \pm 0.17$ & $3.9 \pm 0.16^{*}$ & $4.0 \pm 0.16^{*}$ & $3.6 \pm 0.15$ \\
ECF (\% body weight) & $34 \pm 2.1$ & $37 \pm 1.6$ & $29 \pm 5.5$ & $33 \pm 5.0$ \\
TBW (\% body weight) & $87 \pm 4.3$ & $78 \pm 1.8$ & $67 \pm 4.3^{*}$ & $83 \pm 7.5$ \\
\hline
\end{tabular}

NOTE. $n=8$ patients. Data given as mean \pm SEM.

$* P<.05 \vee$ pre-ECLS value (baseline). 
bypass, this would also suggest an increase in water in the extracellular space.

Physiological sequelae of increased ECF might include increased lung water (and thus pulmonary dysfunction), and generalized tissue edema. Lung field opacification seen on chest $x$-ray (sometimes referred to as "white out") during bypass is thought to be secondary to one or more of several factors: (1) increased pulmonary ECF and interstitial fluid through net capillary filtration; (2) decreased airway pressures as the ventilator is initially weaned upon initiation of bypass, with resultant alveolar collapse, and thus, more dense pulmonary tissue; and (3) differential shunting of transpulmonary blood flow, again with a resultant increase in density of the pulmonary parenchyma. The resolution of this radiographic finding has been found to be associated with lung recovery and decannulation from bypass. ${ }^{9} \mathrm{Al}-$ though opacification may not appear in the chest $\mathrm{x}$-ray of every patient placed on ECLS, modification of "rest" ventilator settings (eg, increased positive end-expiratory pressure) may prevent it. ${ }^{10}$

All patients were pharmacologically paralyzed with pancuronium prior to the institution of ECLS. While there may be a role for pancuronium in edema development, all paralytic agents were discontinued once bypass was initiated, allowing patients to move spontaneously. Most patients were clinically noted to be edematous prior to the initiation of bypass; this edema resolved during the bypass run.

Prior to decannulation from bypass, ECF is significantly lower with a parallel decrease in TBW and body weight, all of which would support an overall negative fluid balance (greater water loss with respect to a decreasing patient weight while on bypass), which is corroborated by the volume decreases seen in Fig 3. At the conclusion of ECLS, the resultant fluid composition was similar in composition to that seen prior to ECLS. Fluid removal during ECLS is typically achieved with the routine use of diuretics or hemofiltration, if necessary, and would explain the significant decrease in TBW and ECF near the conclusion of ECLS. While no patient in the current series underwent hemofiltration during ECLS, all were treated with furosemide during, and in some cases after, the ECLS course.

Several etiologies of neonatal respiratory failure are represented by the patients in this study, including respiratory failure due to persistent fetal circulation, sepsis, and cardiorespiratory failure from a cardiac malformation. All patients were similar in that all had significant respiratory failure unrespon- sive to medical management with an associated high mortality risk. All newborns were placed on ECLS and were decannulated after a successful trial off ECLS, demonstrating evidence of recovered lung function. All patients but one survived to discharge (a late death).

No adverse effects were seen with successive determinations of ECF and TBW. Kazzi et al ${ }^{5}$ reported the death of three of seven 2-month-old lambs in their series from pulmonary failure, possibly related to high serum bromide levels. In our previous studies of infants, serum bromide levels typically peaked at 18 $\mathrm{mg} / \mathrm{dL}$ (range, 7.6 to $36.4 \mathrm{mg} / \mathrm{dL}$ ) while troughs averaged $7 \mathrm{mg} / \mathrm{dL}$ (range, 0 to $20.6 \mathrm{mg} / \mathrm{dL}$ ) when determinations were conducted on a weekly basis. Scrum bromide levels in three of the eight patients presented here averaged peak values of $28.8 \mathrm{mg} / \mathrm{dL}$ (range, 13.6 to $45.2 \mathrm{mg} / \mathrm{dL}$ ) with trough values of 13.7 $\mathrm{mg} / \mathrm{dL}$ (range, 2.9 to $21.0 \mathrm{mg} / \mathrm{dL}$ ). Specific information regarding bromide toxicity in neonates is not known. In the adult human, levels of $50 \mathrm{mg} / \mathrm{dL}$ are associated with a sedative effect, hence the use of bromide compounds as sleeping agents. A level of 150 $\mathrm{mg} / \mathrm{dL}$ is reportedly associated with symptoms of toxicity in the adult, chiefly neuropsychiatric in origin (eg, dysarthria, tremor, stupor, coma). ${ }^{11}$ In the initial four patients studied with this protocol, the serum bromide trough level was assayed prior to each administration of sodium bromide in order to monitor levels, and to avoid peak bromide levels greater than 35 to $40 \mathrm{mg} / \mathrm{dL}$.

In the calculation of ECF, TBW, and corrected patient weight during ECLS, a weight of $0.63 \mathrm{~kg}$ was added to the patient's weight to account for "extracorporeal weight" of the blood volume in the ECLS circuit. This volume was calculated as the mean of the volumes of prime (normal saline, packed red blood cells, albumin, etc) necessary to fill each of 3 newborn circuits $\left(0.8 \mathrm{~m}^{2}\right.$ membrane oxygenator size $)$ prior to clinical use. The addition of this weight to the patient's weight is legitimate because it represents a very real space for distribution of sodium bromide and deuterium oxide. Neglecting this factor in calculating ECF and TBW percentages would result in an overestimation of body fluid compartments because of an incorrectly low body weight. Standardizing changes in calculated ECF and TBW volumes, and patient weights in Fig 3 allow absolute changes in volumes to be discerned, rather than attempting to determine the result of sometimes opposite changes in weight and ECF or TBW. The added weight of 0.63 $\mathrm{kg}$ assumes a $1 \mathrm{~g} / \mathrm{mL}$ density of blood. 
In summary, neonates with respiratory insufficiency have an increased body weight, ECF, and TBW once placed on ECLS. Both TBW and ECF decrease during the ECLS run, which parallel im- provement in lung function. TBW, ECF, and body weight were similar prior to and after decannulation from ECLS. These changes in ECF, TBW, and body weight appear to be associated with lung recovery.

\section{REFERENCES}

1. Bartlett RH: Extracorporeal life support for cardiopulmonary failure. Curr Prob Surg 27:623-705, 1990

2. Coran AG, Drongowski RA: Body fluid compartment changes following neonatal surgery. J Pediatr Surg 24:829-832, 1989

3. Coran AG, Drongowski RA, Wesley IR: Changes in total body water and extracellular fluid volume in infants receiving total parenteral nutrition. J Pediatr Surg 19:771-776, 1984

4. Cleland J, Pluth JR, Tauxe WN, et al: Blood volume and body fluid compartment changes soon after closed and open intracardiac surgery. J Thorac Cardiovasc Surg 52:698-705, 1966

5. Kazzi JK, Schwartz CA, Palder SB, et al: Effect of extracorporeal membrane oxygenation on body water content and distribution in lambs. ASAIO Trans 36:817-820, 1990

6. Brans YW, Cornish JD, Kuehl TJ, et al: Effect of extracorporeal membrane oxygenation on body water content and distribution of baboon neonates. Pediatr Res 20:381-384, 1986
7. Manning PB, Morgan RA, Coran AG, et al: Body fluid compartment changes following cardiopulmonary bypass in dogs. Pediatr Surg Int 3:351-353, 1988

8. Delaney AG, Zapol WM, Erdmann AJ III: Pulmonary transvascular fluid dynamics with extracorporeal membrane oxygenation in awake lambs. Surg Forum 29:194-196, 1978

9. Kelly RE Jr, Phillips JD, Foglia RP, et al: Pulmonary edema and fluid mobilization as determinants of the duration of ECMO support. J Pediatr Surg 26:1016-1022, 1991

10. Keszler M, Subramanian KNS, Smith YA, et al: Pulmonary management during extracorporeal membrane oxygenation. Crit Care Med 17:495-500, 1989

11. Nuki G, Richardson P, Goggin MJ, et al: Four cases of bromism. Br Med J 2:390-391, 1966

\section{Discussion}

Steve Teich (Powell, OH): The initial increase in body weight that occurs in newborns with respiratory failure is a well-known clinical entity. Loss of body weight is associated with resolution of the respiratory failure and subsequent decannulation from ECMO. The authors have attempted to correlate recovery of lung function with fluid shifts occurring simultaneously. I have several questions and a comment.

First, you presented a heterogeneous group of eight patients with a variety of disease processes. Most significantly, two patients in the group were put on ECMO for the treatment of sepsis. Given the fact that sepsis is associated with a capillary leak syndrome and the release of a multitude of vasoactive mediators, shouldn't these patients by excluded from the study?

Second, half the patients in the study had venovenous ECMO, and half the patients had venoarterial ECMO. Did you see any difference in fluid compartment shifts between these two types of ECMO?

Third, you conclude that decrease in total body water and extracellular fluid occurs throughout the ECMO run, and parallels an improvement in lung function. Yet according to the graphs you show, TBW and ECF actually increase significantly for at least 24 hours prior to cessation of ECMO. How do you explain the significance of this phenomenon?

Fourth, other researchers, such as Billie Short in Washington, DC, have demonstrated increased re- nin, aldosterone, and atrial naturitic peptide levels in infants while on ECMO. Did you measure any of these blood levels in an attempt to correlate them with the fluid shifts that you measured?

Finally, I think it will be helpful to perform a laboratory test using a homogeneous group of animals to better answer some of these questions.

David Collins (San Diego, CA): It is misleading to express body water as a percentage of body weight since the majority of body weight consists of water. For example, the body water in absolute amounts could be double but if it starts out as $90 \%$ of body weight it would only go up to $95 \%$, which would not give the true picture of the increase in body weight. Wouldn't the numbers be more meaningful if the water compartments could be related to the dry body weight?

Robert Foglia (St Louis, MI): You show that TBW water decreases but body weight stays high. Why is there a difference? And would you speculate if you think there's an association between body water and pulmonary function. Have you tried to accelerate the fluid normalization with the use of diuretics?

Harry L. Anderson III (response): I'll start with Dr Collins' question first. We spent some time deciding which format would best express the changes. The only common term we had with each measurement was the weight of the patient at that particular slice in time, and that's typically how we have performed 
measurements of patients receiving parenteral nutrition-so we tried to keep the same convention. We felt it best not to select one initial weight and try to maintain that throughout the different measurements.

The other conflicting variable-and this goes back to Dr. Teich's questions-is that there is one intervention not present in all measurements, and that variable is the presence or absence of the extracorporeal circuit and its additional blood volume, with regard to added extracellular fluid, body water, and weight. The circuit volume is not present before or after bypass, when the measurements of weight, ECF, and TBW were found to be the same. In actuality then, the extracorporeal volume weight must be added to the patient weight while on bypass, so that you lose this reference point with the step of prebypass to on bypass, yet still direct comparison is difficult.

These decreases in ECF and TBW may actually be starting from a truly higher reference point. During bypass, there is a noticeable drop in TBW and ECF. After decannulation, the post-ECMO measurement shows the result of these changes, now without the added extracorporeal circuit volume-these values are now the same as they were prior to bypass.

Dr Foglia asked about accelerating fluid loss during ECMO-all patients underwent a diurctic-induced forced diuresis or hemofiltration, which is part of our normal protocol.

In terms of our heterogeneous group of patients, all patients were more or less moribund prior to the institution of ECMO, both septic and nonseptic patients alike. We collected data from all eight patients and presented them here as a group. We didn't see any differences in venovenous versus venoarterial bypass treated patients; therefore, these groups were not presented separately.

One parameter that we definitely found to increase over prebypass levels was ECF space. Also in response to Dr Teich, we did not specifically measure renin, aldosterone, or atrial natriuretic factor during the bypass runs in these patients.

In terms of an animal model, Brans and Klein looked at similar parameters during both short-run and long-run bypass in sheep, and were not able to document any changes in ECF and TBW during experimental bypass support. 